\title{
Human Health Risk Management in Engineered Nanomaterials (ENMs): the Case of Carbon-Based Nanomaterials
}

\author{
EP Koumoulos and CA Charitidis* \\ Department of Materials Science and Engineering, National Technical University of Athens, Greece
}

Submission: May 09, 2017 ; Published: June 07, 2017

*Corresponding author: CA Charitidis, Department of Materials Science and Engineering, National Technical University of Athens, Greece, Email: charitidis@chemeng.ntua.gr

\section{Introduction}

There is no doubt regarding the importance and opportunities of engineered Nanomaterials (ENM). The anticipated benefits of ENM applications resulted in expenditure of $\$ 18$ billion worldwide on nanotechnology research and development in 2008. They already are commonly employed in electronics, photovoltaic, catalysis, cosmetic industry, medicine and pharmacy. Nanotechnology applications will contribute positively to the quality of life through the production of light and durable materials, cleaner energy and inexpensive clean water production, as well as by enabling several beneficial medical applications, especially smart drugs. Additionally, great environmental benefits are predicted from nanotechnologyrelated applications because of the savings in raw materials, the consumption of natural resources and a reduced environmental pollution.

Some of the properties that make ENM so unique and beneficial for technological applications may also endanger human health through the potential induction of cyto and genotoxic effects, inflammation and even cancer. Therefore scientists, governmental and non-governmental organizations, as well as consumers express concern about potential risks to human health and the environment posed by rapidly emerging nanotechnology applications. Exposures to engineered nanoparticles (ENPs) either through contact with consumer products or air, water and food sources, either through contact in the production process are an emerging potential threat to human health. Nanoparticles can access the human body through the main ports of entry such as the skin, the lungs, the gastrointestinal tract, but also through fine capillaries, travel through the body and interact with organs [1].

An epidemiological feasibility study of CNT workers initiated in 2008 revealed most manufacturers were small companies that had no environmental/occupational health and safety person and little knowledge about this topic. By 2015, the global market for nanotechnology-related products employed 2 million workers to support nanotechnology manufacturing, and $\$ 1$ trillion in sales of nanotechnology-related products. Risk assessment and risk management for the production and the use of engineered nanomaterial products are a necessity today with four main steps being hazard identification, hazard characterization, the assessment of exposure and the analysis of transport processes.

Based on these a final risk assessment has to be made. Risk assessment integrates the results of the four steps of the risk assessment process and aims at assessing the likelihood of occurrence of a given hazard in a certain exposure situation. The ultimate goal of current risk assessment paradigms is to be able to provide quantitative predictions of given risks enabling evidence-based risk management that is based on quantitative assessment of a given risk in a population. The need for risk assessment of ENM has generated also a need for a novel risk assessment concept. Even though the keysteps of risk assessment, notably hazard identification, hazard characterization and exposure assessment followed by risk characterization, in an optimal case leading to quantitative risk assessment remains the cornerstone of assessment of safety of ENM, special features of ENM require modifications to the current procedures [2].

However, there are several additional datasets that require special attention and availability of which is essential for the reliable risk assessment of ENM. Main difficulty in risk assessment of engineered nanomaterial is that there is no uniform group of substances. Applications of engineered nanoparticles are for example silver (Ag NP), carbon based materials, titanium dioxide, zinc oxide, gold and other with very different characteristics of toxic potential. Nanowerk identified 2500 commercial 
nanomaterials, including $\sim 27 \%$ metal oxides, $24 \%$ CNTs, $18 \%$ elements, $7 \%$ quantum dots, and $5 \%$ fullerenes. There is literature available providing important background knowledge on the toxic potential of various substances, but it is still largely unknown which properties determine and influence the toxicity of particular nanoparticles. With newly identified or emerging health risks covering novel or emerging technologies, the use of a great caution or precautionary principle is usually justified in the early stages of risk assessment.

In such cases, risks assessments suffer from lack of data. This approach is emphasized for example by the European Union Scientific Committee on Emerging and Newly Identified Health Risks or Scientific Committee on Consumer Safety (SCCS) when dealing the evaluation of ENM with very limited amount of data (SCCS, 2008). This approach has also been characterized in the context of risk assessment and management by the statement "no data, no market" (SCCS, 2008). When the amount and quality of data are improved, chances to make more accurate risk assessments are also improved, and the importance of emphasizing uncertainties is consequently reduced. In European Union REACH legislation is the current regulatory framework for chemical risk assessment and management, and it also applies to ENM [3]. One unique risk assessment challenge associated with ENM is the entry of a huge number of these qualitatively new compounds to be tackled with the current risk assessment process, a challenge so large that the existing approach cannot deal with it. Therefore novel but reliable enough approaches need to develop.

Even though REACH quite thoroughly considers the physicalchemical characteristics of ENM under substance definition within the legislation (REACH, 2006), the understanding of the true nature of ENM and the correlation of ENM characteristics and biological effects is not fully characterized. REACH also characterizes the remarkable speed of the development of nanotechnologies and impact on the volume of production of ENM and the rapidity of adaptation of these technologies in consumer products. For example, the word 'nano' has not been mentioned in the legislation rendering its capability to cover risk assessment issues relevant ENM and nanotechnologies a challenge, in spite the fact that legislation came into force in 2006 (REACH, 2006). The Nano Commission of the German government has also identified some key issues of concern of ENM dealing with the existing data gaps and contributing to a risk assessment of ENM.

The identified major issues included:

a. Consideration of high production volume ENM;

b. High mobility in different media

c. Persistency of the nano characteristics

d. Potential to bioaccumulate

e. High reactivity f. Possible interactions with other toxicants

g. Interaction reactions especially at the nanoparticle surface over time

\section{h. Challenges in ENM characterization \\ i. Distribution of ENM}

Perhaps the most demanding challenge for risk assessment is the need to develop an intelligent, tiered testing strategy aided by carefully designed decision tree approach to be able reduces the amount of resources for ENM risk assessment without jeopardizing the safe use of these unique materials. Meeting these challenges would also greatly benefit assessment of risks of other materials.

Other discoveries like truly self-cleaning, non-stick, highly scratch-resistant surfaces, intelligent paints, etc., went through similar rapid evolution. It has become a new challenge for industry to adjust to the rapid speed of product development based on nanotechnology in order to be able to adopt innovations in nanotechnology at a similarly rapid speed. In some cases it can mean that an entire manufacturing process or product line will no longer be competitive if it is not adjusted in time. It also means that it has become essential for industry to rapidly learn the language of other industries and disciplines so it can assess emerging competitors, sometimes from a completely different field, and to spot developments that can be adopted and those that may dig into one's own market share in the future.

The volume of ENMs being used for commercial applications increases year by year. The scientific community tries to investigate the potential human health hazards from exposure to ENMs. The risks of this versatile class of materials have to be assessed in order to manage them properly. If it is desirable to extend the use of nanotechnology and especially, to broaden the applications of ENMs, we should gain their benefits with minimal risk, taking into account the safest alternatives for each particular application. It is clear, that every material has benefits, but also reserves risks, which need the proper corrective actions in order to eliminate them. Both government and industry should drive these actions. Risk management seems to be obligatory as the mass production of ENMs has already been established. At this point, it should be mentioned that risk management refers not only to the estimation of risks of ENMs, but also to the elimination of the uncertainty of the assessed risks.

It is truth, that there is still a lot of uncertainty concerning the safe handling and the risks on health of ENMs. For this reason, ENMs should be considered as substances of high concern unless, or until, sound evidence will show otherwise. The foremost barriers for conducting risk assessment about ENMs, except for those have been discussed above, are the lack of agreement of an appropriate metric system for assessing the exposure and the default of ENM-specific toxic endpoints. 
Moreover, the particularly characteristics of ENM that could be utilized for risk management of ENM are still under investigation. Selection of the most relevant metric(s) (e.g. surface area, particle count/per particle size, particle mass, and particle charge [3] for health-related sampling of ENM is an important component in the development of the concepts, methods and technology for ENM monitoring at workplaces and for the correlation with observed toxic effects [4-5].

Among others, some authors have recommended surface area, as a better metric $[4,6,7]$. However, there is neither the appropriate practical equipment for measuring the surface area nor measurement protocols or baseline measurements. For this reason, there is a need to develop not only efficient online measurement devices that will be able as well to identify airborne nanoparticles in workplace but also lower-cost, so as to be affordable enough to the employers [8].

Many difficulties appear in comparing the toxicity results of carbon nanomaterials, due to the different and unique structures of them. It is obvious that differences in opinions about the potential hazards of exposures to CNTs and CNFs exist. The various types of CNTs and CNFs, in the range of 50,000 [9], render the risk assessment of these materials challenging [10]. All the parameters that seem to affect the toxicity performance of CNTs and CNFs (diameter, length, surface area and functionalization) should be examined.

The available experimental models for toxicity testing should also be reconsidered, in order to have more realistic results. Moreover, it is important to develop computational models to predict the toxicity of CNTs and CNFs as well as to determine their release mechanisms in the environment. However, the lack of reliable experimental data slows down the development of these models. Finally, there is an urgent need for the scientific community not only to associate the nanoproducts' development with suitable processes for assessment, monitoring, managing and reducing the posed risks of ENMs to human health and the environment, but also to disseminate these efforts efficiently, in order to win public acceptance.

\section{References}

1. Gajewicz A, Rasulev B, Dinadayalane TC, Urbaszek P, Puzyn T, et al. (2012) Advancing risk assessment of engineered nanomaterials: application of computational approaches. Adv Drug Deliv Rev 64(15): 1663-1693.

2. Savolainen K, Alenius H, Norppa H, Pylkkanen L, Tuomi T, et al. (2012) Toxicol 269: 92

3. K Aschberger, C Micheletti, FM Sokull-Kluttgen, B Christensen (2011) Analysis of currently available data for characterising the risk of engineered nanomaterials to the environment and human health-lessons learned from four case studies. Environ Int 37: 1143-1156.

4. CL Tran, D Buchanan, RT Cullen, A Searl, ADJones, et al. (2000) Inhalation of poorly soluble particles. ii. Influence of particle surface area on inflammation and clearance. In hal Toxicol 12: 1113-1126.

5. KE Rushton, J Jiang, SS Leonard, S Eberly, V Castranova, et al. (2010) J Toxicol Environ Health A 73: 445-461.

6. G Oberdorster (2010) Safety assessment for nanotechnology and nanomedicine: concepts of nanotoxicology. J Intern Med 267: 89-105.

7. T M Sager, V Castranova (2009) Surface area of particle administered versus mass in determining the pulmonary toxicity of ultrafine and fine carbon black: comparison to ultrafine titanium dioxide. Part Fibre Toxicol 6:15.

8. DA Maynard, RJ Aitken (2007) Assessing exposure to airborne nanomaterials: Current abilities and future requirements. Nanotoxicology 1:26-41.

9. P Schulte, MK Schubauer Berigan, C Mayweather, C L Geraci, R Zumwalde (2009) J Occup Environ Med 51: 323-335.

10. Costas A Charitidis (2016) Risk management of engineered nanomaterials in EU-The case of carbon nanotubes and carbon nanofibers: A review. Trans Mat Res Soc Japan 41(1): 1-11.

\section{Your next submission with Juniper Publishers will reach you the below assets}

Commons Attribution 4.0 Licens DOI: 10.19080/JOJCS.2017.03.555609 